\title{
STUDIES ON PATHOGENESIS AND RECOVERY IN ERYSIPELAS
}

\author{
By THOMAS FRANCIS, JR.
}

(From the Department of Internal Medicine, Yale University School of Medicine and the Medical Service of the New Haven Hospital, New Haven, Conn.)

(Received for publication June 27, 1928)

During the past three years Birkhaug (1-5) has reported the results of an extensive investigation of many phases of erysipelas both in experimental animals and in man. His observations may be summarized briefly. Hemolytic streptococci from erysipelas were found to form an immunologically specific group of streptococci. Ninetyone per cent of such organisms tested by the agglutinin absorption technique fell into this group. No cross agglutination with Streptococcus scarlatinae was noted (1). Serum obtained by immunizing rabbits against strains of erysipelas streptococci protected the skin of normal rabbits from infection with the homologous and heterologous erysipelas strains when the serum and bacteria were injected together. Passive immunity was also obtained by intravenous or subcutaneous injection of the serum twenty-four hours before the inoculation of a supralethal dose of organisms. Under these conditions the results with heterologous organisms were not so regular as with the homologous strains (2). The immune serum caused a blanching of the erysipelatous lesion in patients or tended to prevent its spread. This effect was not obtained with scarlatinal antitoxin; nor was blanching of the scarlatinal rash obtained with anti-erysipelas serum. Blanching of the erysipelatous lesion was also obtained with convalescent erysipelas serum (2). Broth cultures of erysipelas streptococci uniformly gave toxic filtrates which in dilutions of 1:1000 produced a skin reaction in susceptible individuals similar to a positive Dick test. Some strains produced filtrates which contained 30,000 to 50,000 S.T.D. of toxin per cubic centimeter. It was noted that the maximum production of toxin was obtained with 48 hours' incubation and that a 
definite decrease in the amount of toxin occurred after 90 hours. The cutaneous reaction produced by 1 S.T.D. could be completely neutralized "by mixing the skin test dose with an equal amount of convalescent erysipelas serum, or with $0.001 \mathrm{cc}$. of erysipelas antistreptococcic rabbit or donkey sera" (3). Eighteen patients with erysipelas gave positive reactions to 1 S.T.D. of toxin on admission to the hospital and as the erysipelatous lesion cleared and convalescence was established the reactivity of the skin to a similar dose was lost. This occurred from 5 to 38 days after the original test. During the acute stage of erysipelas when skin reactions were positive there was demonstrable in the patient's serum a toxic substance which caused a local cutaneous reaction in normal susceptible individuals. This reaction could be neutralized by immune or convalescent erysipelas serum. A toxic substance occurred in large amounts in the urine. With the disappearance of the cutaneous susceptibility to the toxin, following recovery from the disease, the presence of antitoxin in the serum of patients was demonstrated by means of toxin neutralization tests in the skin of susceptible individuals. Cross neutralization of the erysipelas toxin by scarlatinal antitoxin was not obtained (3). Anti-erysipelas donkey or rabbit serum produced favorable therapeutic results when given early in the disease. The impression obtained was that the serum was antitoxic in nature. The amount of serum required was determined by the inoculation of 1 S.T.D. of toxin into the skin of the patient simultaneously with the intramuscular or intravenous administration of the therapeutic serum. A positive or negative reaction was considered to indicate incomplete or complete neutralization of the circulating toxin, respectively (4). In patients with recurrent attacks of erysipelas the presence of antitoxin in the blood and the insusceptibility of the skin to the toxin were replaced after an interval by the return of skin sensitivity and the absence of antitoxin in the circulating blood. Active immunization by means of the toxic filtrate was considered to be highly efficacious in preventing recurrences. In the group of cases reported, immunization caused a decrease in the frequency of recurrences, induced a loss of skin reactivity and increased the neutralizing capacity of the patient's serum (5). In normal individuals tested, 21 per cent of 272 school children, ranging in age from 7 to 17 years, and 27 per cent of 135 hospital 
patients (other than those with erysipelas), of ages from 18 to 72 years, gave positive skin reactions to 1 S.T.D. of toxin (3).

Kaplan and Singer (6) also noted disappearance or diminution of the cutaneous reactivity during convalescence from the disease. With an antitoxin produced by repeated injections of culture filtrate of erysipelas streptococci into human subjects they obtained complete neutralization of the homologous undiluted toxin in 73.7 per cent of the susceptible individuals in whom tests were made, whereas, with convalescent erysipelas serum it was obtained in only 38.1 per cent. Definite cross neutralization of the toxin by scarlet fever antitoxin and of scarlet fever toxin by the erysipelas antitoxin or convalescent erysipelas serum was noted. Blanching of the erysipelatous lesion occurred in $\mathbf{5 0}$ per cent of those tested with the authors' antitoxin, but was uniformly absent when convalescent erysipelas serum was used.

As Birkhaug has pointed out (5), the results of his studies on erysipelas present a striking parallelism with the observations on scarlet fever which have been reported during the last few years by Dick and Dick (7), Dochez (8), Blake and Trask (9), and numerous others; so much so, in fact, that one would be led to believe that in its pathogenesis erysipelas is essentially comparable to scarlet fever, and, consequently, amenable to treatment by a specific method similar to that which has proved successful in the case of the latter disease. But on clinical grounds this parallelism does not obtain. Scarlet fever is most prevalent between the ages of six months and twenty years; erysipelas is most prevalent under six months and over twenty years. An attack of scarlet fever almost invariably gives a lasting immunity; one attack of erysipelas would appear to render many individuals, at least, more prone to subsequent attacks. It is also noteworthy that recurrent erysipelas tends to involve the same areas affected in previous attacks. In scarlet fever the essential and most evident clinical phenomena are removed from the local focus of infection and have been shown to be due to a toxin absorbed from the local focus; in erysipelas the essential and most evident phenomenon is the local spreading lesion of the skin due to actual infection of the skin by streptococci and dispersed specific lesions comparable to the toxic lesions of scarlet fever are not present. Clinically, scarlet fever pre- 
sents three relatively distinct phases: the early toxic phase, the septic phase, and the late sequelae. In its pathology and its clinical aspects erysipelas would appear to simulate more closely the septic than the toxic phase of scarlet fever. Like scarlet fever, of course, it may be followed by late sequelae.

Inability to correlate satisfactorily the experimental observations of Birkhaug with the well established clinical phenomena of erysipelas has led to the present study. Three aspects of the subject have been investigated: (1) the reactivity of the skin of erysipelas patients to intracutaneous injections of filtrates of erysipelas streptococci; (2) the presence of a toxic substance in the blood of patients acutely ill with erysipelas; and (3) the capacity of the serum of erysipelas patients to neutralize the toxic action of culture filtrates.

\section{SKIN REACTIVITY OF PATIENTS WITH ERYSIPELAS TO FILTRATES OF ÉRYSIPELAS STREPTOCOCCI}

The skin reactions to sterile filtrates from broth cultures of erysipelas streptococci were studied in 30 patients with erysipelas at intervals during the acute and convalescent stages of the disease. With one exception the patients were all on the adult medical wards of the New Haven Hospital, their ages ranging from 13 to 70 years. The filtrates employed in most instances were prepared in our laboratory from cultures of hemolytic streptococci cultivated directly from the lesions of typical cases of erysipelas. For comparison a standardized erysipelas toxin obtained from the Squibb Laboratories through the courtesy of Dr. J. F. Anderson was used in some of the cases.

Preparation and standardization of filtrates. Flasks containing 250 cc. of 1 per cent defibrinated rabbit's blood, buffered, meat infusion broth, $\mathrm{pH} 7.4$ to 7.6 , were inoculated with $2.5 \mathrm{cc}$. of an 18 hour broth culture of erysipelas streptococci. After incubation for 48 hours at $37^{\circ} \mathrm{C}$. the culture was passed through a Berkefeld filter, the filtrate tested for sterility and bottled. The filtrates were standardized in terms of skin test doses per cubic centimeter by injecting $0.1 \mathrm{cc}$. of graded dilutions in the skin of the most reactive individual available. One-tenth cubic centimeter of that dilution which caused a local erythema approximately $10 \mathrm{~mm}$. in diameter 24 hours after injection 
was considered to be 1 S.T.D. Titrated in this way the filtrates were found to contain from 2,000 to 3,000 S.T.D. per cubic centimeter. The Squibb toxin had been standardized by the makers, $1 \mathrm{cc}$. containing 5,000 S.T.D. One-tenth cubic centimeter of a 1:500 dilution of this toxin caused a positive reaction approximately $10 \mathrm{~mm}$. in diameter in the same subject used in the standardization of our filtrates. Negative heat controls were obtained only by boiling the filtrates two to four hours.

Technique of skin tests. Graded dilutions of filtrate containing from 1 to 50 S.T.D. of toxin in $0.1 \mathrm{cc}$. volume were injected intradermally on the flexor surfaces of the forearms, the doses employed being 1,3 or 5,10 , and 30 or 50 S.T.D. The complete series of doses was not used in each patient. In 12 cases 10 S.T.D. was the largest dose employed, while in a moderate number 3 S.T.D. was the smallest dose used, and in a few an intermediate dose was omitted. In general, the same doses were used in the same patient throughout the course of his illness. Heat controls for the largest doses, at least, were used. Readings were made at 24 hours and the size and intensity of the reaction recorded. All reactions which were less than $6 \mathrm{~mm}$. in diameter were considered negative.

The results of the observations on the skin reactivity of erysipelas patients to erysipelas streptococcus culture filtrates are shown in chart 1 , in which the patients are divided into two groups according to the degree of reactivity at the time of the first skin test and arranged within the groups according to the duration of the disease. The results are recorded so as to show the smallest dose to which each patient reacted at each test. A positive reaction to 30 or 50 S.T.D. but not to smaller doses is recorded \pm ; to 10 S.T.D. but not to smaller doses, +; to 3 or 5 S.T.D. but not to 1 S.T.D., \pm \pm ; to 1 S.T.D., ++ . Failure to react to any dose is recorded - . The degree of reaction to any one of the individual doses is indicated by the size and intensity of the symbol. The duration of the disease in each patient is shown by the heavy staggered line which marks the return of the temperature to normal. When this line is broken, the patient had a recurrence or developed a complication. The reactions to heat controls are not included, since it was found that the reaction was of about the same degree as that to the unheated filtrate. 
The results as charted demonstrate that 20 cases (Group I) showed on the first test during the active stage of the disease either no reaction or only a very slight degree of reactivity of somewhat doubtful

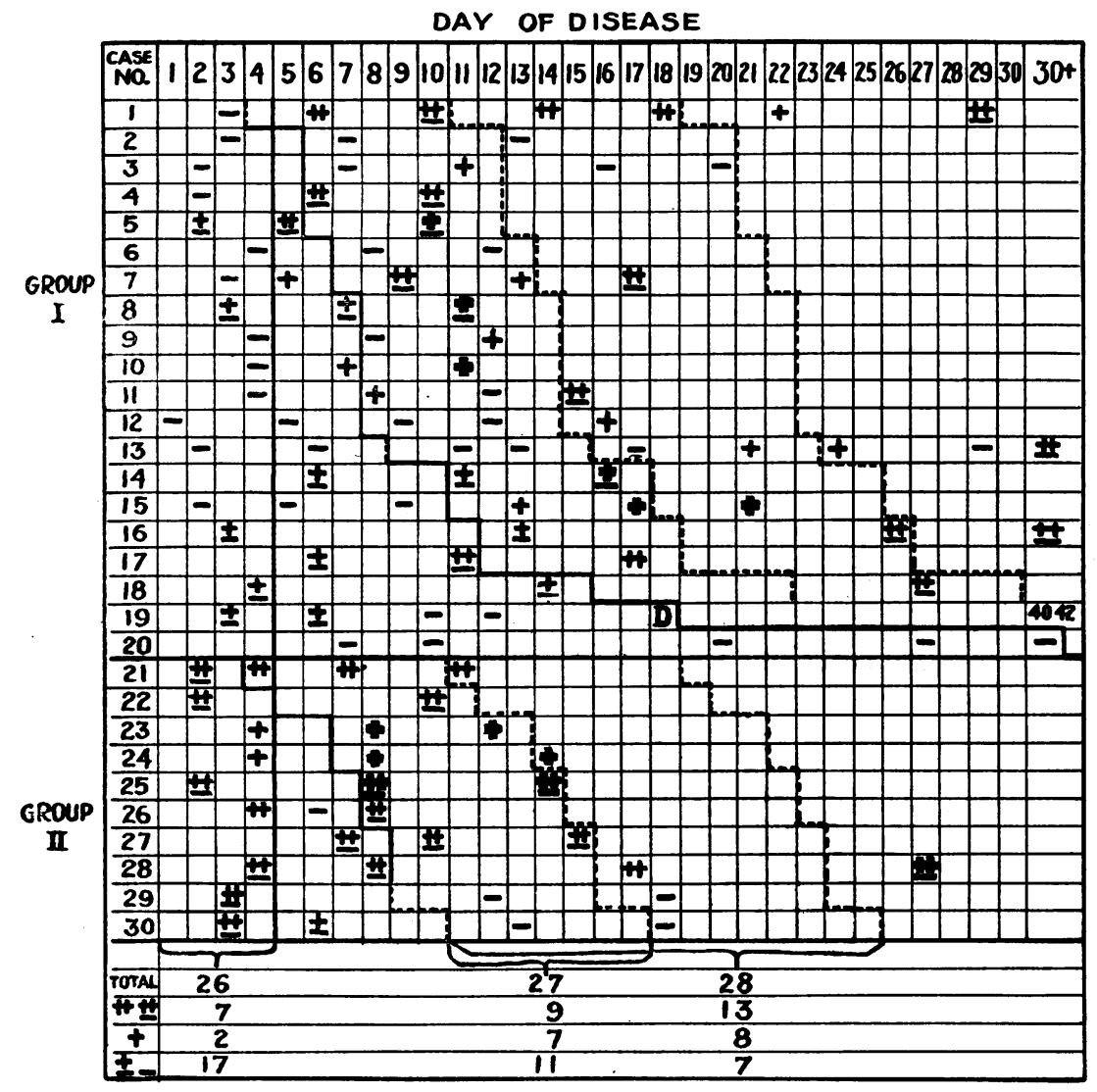

Chart 1. Skin Reactivity of Erysipelas Patients

$-=$ non-reactive $; \pm=$ reactive to 30 or 50 S.T.D.; $+=$ reactive to 10 S.T.D.; $\pm \pm=$ reactive to 3 or 5 S.T.D.; $++=$ reactive to 1 S.T.D. The broken staggered lines mark the end of 7 and 15 day periods, respectively, after return of temperature to normal.

significance, since the 30 to 50 S.T.D. required the use of a 1:10 dilution of the filtrate. They furthermore show that 10 cases (Group II) possessed some degree of reactivity. Of the patients in this group 2 
reacted to 10 S.T.D. but not to less, 7 to 3 or 5 S.T.D., and only 1 to 1 S.T.D. As shown at the bottom of the chart only 7 of the 26 patients tested during the first four days of the disease, at a time when susceptibility to the filtrate should be greatest according to Birkhaug, were sufficiently susceptible to give a positive skin reaction to 3 or 5 S.T.D., 2 more were sufficiently susceptible to react to 10 S.T.D., and 17 were highly insusceptible.

The repeated tests in the later acute period of the disease and during convalescence show a general, though not uniform, tendency for the reactivity of the skin to increase rather than to decrease and become negative as reported by Birkhaug. This is particularly conspicuous in Group I. Between the fourth day of the disease and the end of the first week of convalescence cases $1,4,5,7$, and 17 became reactive to 1,3 , or 5 S.T.D., cases $3,9,10,11$, and 15 to 10 S.T.D., and cases 8 and 14 showed a more marked reaction to 30 and 50 S.T.D., respectively. The relatively fewer observations later in convalescence show that cases $12,13,16$ and 18 eventually became reactive, and that cases 11 and 15 were more susceptible to the filtrate than they had been on earlier tests. Case 3, on the other hand, lost the moderate degree of reactivity exhibited toward the end of the first week of convalescence. In Group II all but 3 cases (26, 29, and 30) either maintained their original reactivity or showed increased susceptibility in the convalescent period. In the whole series of cases there are only three that remained completely negative throughout,- cases 2 and 6 , who were not retested late in convalescence, and case 20 , who ran a prolonged course of migrating erysipelas complicated by metritis, arthritis and sepsis and was not retested after her final recovery on the forty-second day. There were also three cases which showed a diminishing reactivity with the progress of the disease. Case 19, slightly sensitive early in the disease, subsequently became completely non-reactive concurrently with the development of a complicating and fatal sepsis. Cases 29 and 30, one of whom developed a complicating abscess, the other recurrent erysipelas with sepsis, also became non-reactive, though originally susceptible to 5 and 3 S.T.D., respectively. There are four cases which showed a negative skin test between two positive tests. Occasional irregularities in tests of this type are to be expected and their occurrence would not appear to invalidate the general 
relationship between the stage of the disease and the degree of susceptibility to the filtrate exhibited by the study as a whole.

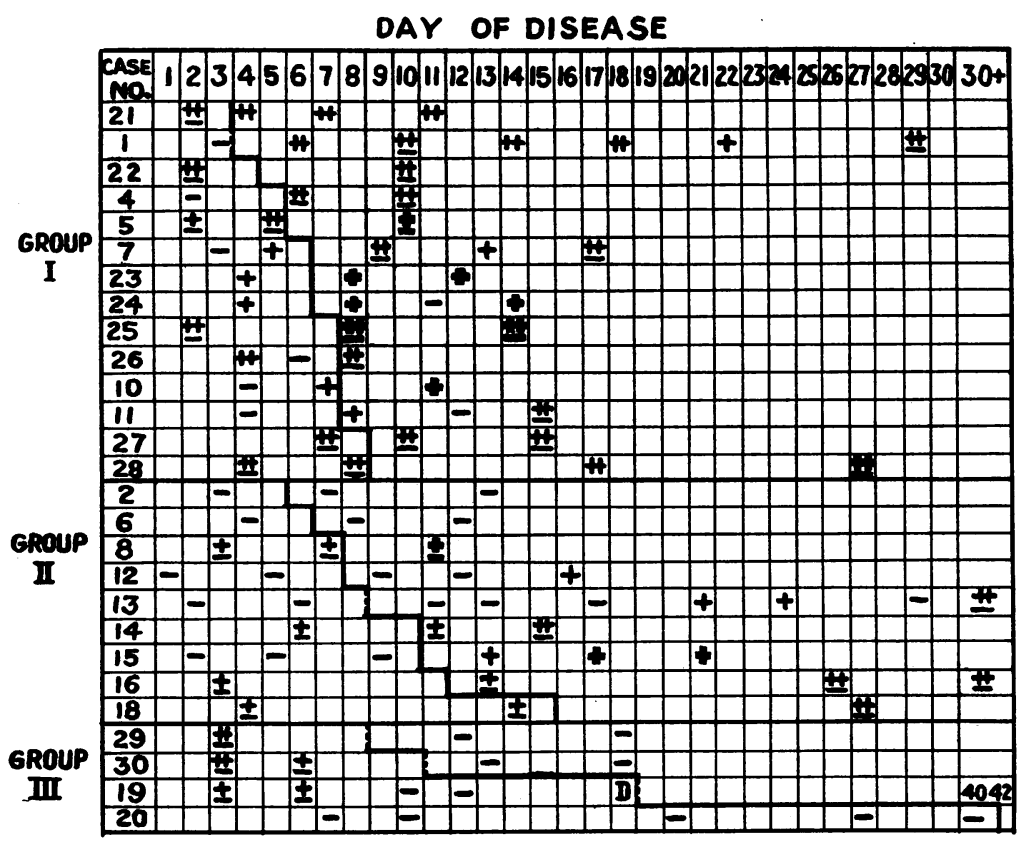

Chart 2. Relationship of Skin Reactivity to Clinical Course of ERYSIPELAS

Case 1. Readmission of Case 13. Recurrence on 8th day of 24 hour's duration. Case 13. Recurrences on the 20th and 29th days both of which lasted approximately 48 hours. Case 29. Abscess developed about left eye on 8 th day and was incised on the 12th day. Case 30. On the 16th day patient had recurrence over entire area previously involved and at that time a blood culture yielded hemolytic streptococci. The recurrence lasted 36 hours. Case 19. Abscesses developed on arms beginning on the 7th day. Hemolytic streptococcus septicemia developed on the 13th day. Exitus on the 18th day. Case 20. Migrating erysipelas with metritis, arthritis and septicemia complicating pregnancy and the puerperium. Recovery on 42 nd day.

In chart 2 the cases have been rearranged into three groups in order to bring out more clearly the relationship between the skin tests and the course of the disease. In Group I are the 14 cases which showed 
positive skin reactions to 10 S.T.D. or less by the eighth day of the disease and maintained positive reactions throughout convalescence. In all of these the duration of the disease was eight days or less, the average duration being 5.9 days. In Group II are the nine cases showing negative skin tests to 10 S.T.D. up to the twelfth day of the disease inclusive. Five of these had a duration of eight days or more, the average duration being 8.8 days. In Group III are the three cases which showed decreasing skin reactivity and case 20 which showed negative tests throughout a long period. All four of these cases developed septic complications with positive blood cultures in three and a fatal outcome in one.

\section{TOXIN IN THE BLOOD OF ERYSIPELAS PATIENTS}

Samples of blood were collected from a series of patients with erysipelas at frequent intervals during the course of the disease. The blood was allowed to clot, centrifuged, and with sterile precautions, the serum was pipetted off and bottled. The initial samples, taken early in the acute stage, were tested for the presence of a toxin by injecting 0.1 or $0.3 \mathrm{cc}$. of the serum into the skin of normal individuals and patients known to be reactive to erysipelas streptococcus culture filtrates. Of 29 such sera tested only 3 caused positive local reactions with any constancy, while 4 others produced a reaction on one occasion, although repeatedly negative on other tests: From these results it would appear very improbable that the majority of patients with erysipelas have any demonstrable or significant amount of specific toxin in the circulating blood during the acute stage of the disease, at least in amounts equivalent to 3 to 10 S.T.D. per cubic centimeter.

NEUTRALIZATION OF CULTURE FILTRATE BY THE SERUM OF ERYSIPELAS PATIENTS

In order to study the development of an antitoxin in the blood of patients recovering from erysipelas, neutralization tests were done with the serial sera obtained from 27 cases of erysipelas of varying duration and severity. Twenty-three of these were patients in whom the skin reactivity was studied and reported upon above.

Technique of neutralization tests. To $0.5 \mathrm{cc}$. of each serum from each 
patient 10 S.T.D. of erysipelas streptococcus culture filtrate in a volume of $0.5 \mathrm{cc}$. was added, so that $0.1 \mathrm{cc}$. of the mixture contained 1 S.T.D. of the filtrate and $0.05 \mathrm{cc}$. of serum. With the last of the series of sera from each patient an additional mixture of a comparable amount of the serum and of a heated filtrate was made. The mixtures

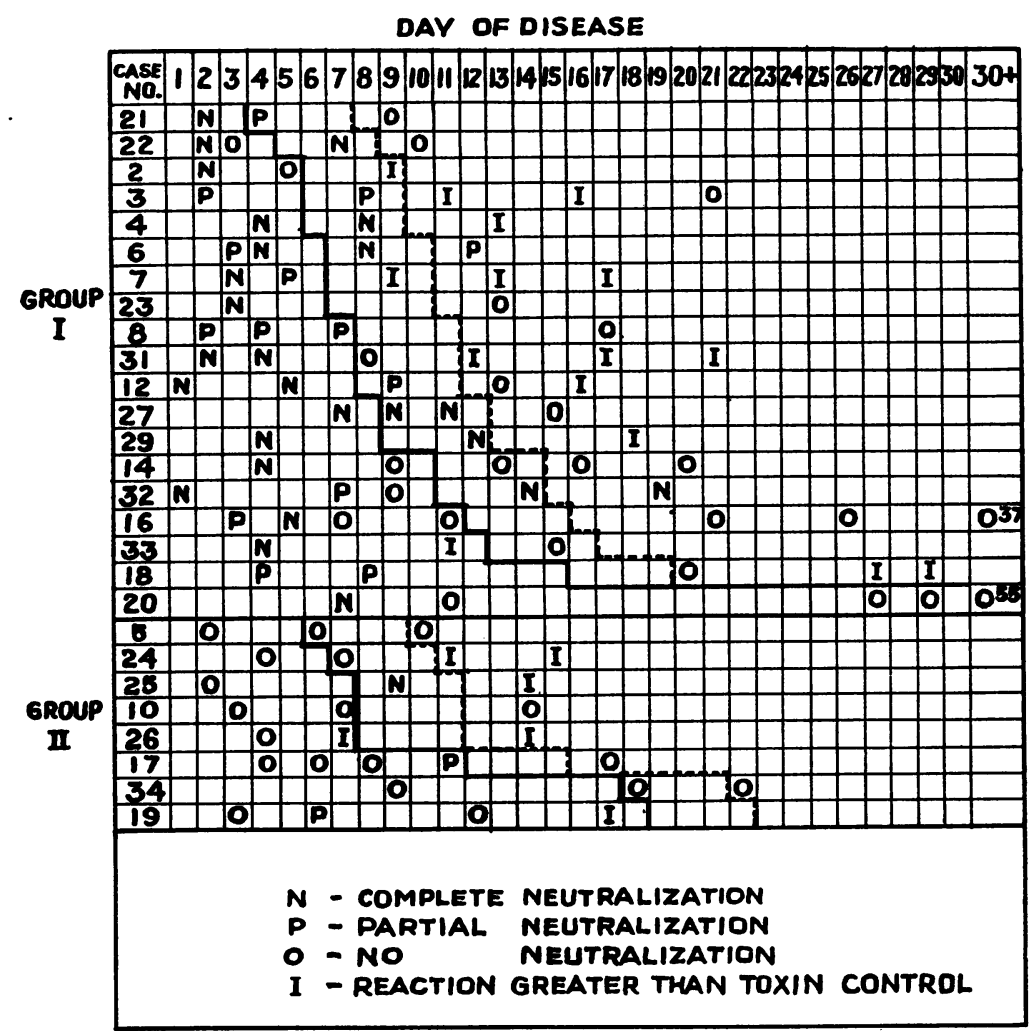

Chart 3. Neutralizing Capacity of the Serum of Erysipelas Patients Case numbers up to 27 agree with those of charts 1 and 2

were thoroughly shaken and heated at $37^{\circ} \mathrm{C}$. for one hour. Onetenth cubic centimeter of each mixture was injected into the skin of individuals reactive to 1 S.T.D. of filtrate. In addition 1 S.T.D. of the filtrate alone was injected at the same time for comparison. 
The reactions were observed and measured at 8,18 , and 24 hours. It was found that the most satisfactory time for recording the results was at 24 hours, and this was adhered to throughout.

The results of the neutralization tests are presented in chart 3 , in which the patients have been divided into two groups according to whether the serum from the early bleedings neutralized or not. The duration of the acute stage of the disease in each case is indicated as in the previous charts. The results obtained from the mixture of the last serum from each patient and the heated filtrate are not recorded, since these uniformly failed to cause a reaction, nor are the reactions to the filtrate alone included since these were uniformly positive.

From chart 3 it will be seen that the great majority of the cases fall into the first group, in which the sera collected early in the acute stage of the disease completely or partially neutralized the toxic action of the filtrate, and that in only 8 cases (Group II) did the early bleedings fail to neutralize. Another very striking result is the failure of the late convalescent sera to neutralize at all. In only 2 cases (Numbers 6 and 32) did convalescent sera collected more than four days after return of the temperature to normal show any capacity to neutralize the filtrate. Not only was there a failure of the convalescent sera to neutralize, but in many instances a more marked reaction occurred than with the filtrate alone. It is furthermore noteworthy that there is no case in which the early sera failed to neutralize and the late convalescent sera neutralized. As with the skin tests, there are a few apparently irregular results, but this is not surprising in view of the nature of the observations.

Correlation of the initial skin and neutralization tests fails to show any consistent relationship. While it is true that 11 of the patients whose sera completely or partially neutralized the filtrate failed to react to 10 S.T.D. of the filtrate, there are 5 cases, whose sera neutralized, who reacted to 10 S.T.D. or less. Of the 7 patients whose early sera failed to neutralize, 3 gave positive skin reactions to 10 S.T.D. or less, but 4 failed to react to 10 S.T.D. It is obvious, therefore, that no simple explanation of the skin reactions on the basis of the presence or absence of a neutralizing antitoxin in the blood is satisfactory in interpreting the results obtained. 


\section{DISCUSSION}

The three important phenomena brought to light in the preceding observations, namely, the tendency for the cutaneous reactivity of erysipelas patients to become more marked during convalescence. the absence of a demonstrable toxin in the circulating blood of patients in the acute stage of the disease, and the neutralization of erysipelas streptococcus culture filtrates by the serum of most patients in the acute phase of the disease with apparent loss of this power during convalescence, fail to support Birkhaug's concept of erysipelas as a specific toxemia, recovery from which is due to the development of an antitoxic immunity.

The results of the skin tests suggest, rather, that an increasing sensitiveness or allergy to streptococcus products, presumably liberated at the site of the erysipelatous lesion, develops with the progress of the disease to convalescence and recovery. The fairly definite correlation between the rapidity with which skin reactivity developed and the clinical course of the disease, as shown in chart 2, furthermore suggests that the development of tissue allergy may play at least a part, perhaps an important part, in the mechanism of recovery from the infection. That the skin reactions were neutralizable not only in sensitive normal individuals but also in sensitive patients in the convalescent period, as proved to be the case on a number of tests, is not surprising, since it has been shown by Dochez and Stevens (10) that the skin reactions of rabbits rendered allergic to undiluted culture filtrates of erysipelas streptococci, are neutralizable during the early phase of sensitivity.

The negative results of the tests for toxin in the circulating blood early in the disease indicate that a specific toxemia in the sense in which it has been shown to occur in scarlet fever (9), plays little or no significant part in erysipelas. This view is further supported by the neutralization tests, in which it is shown that the majority of adult patients with erysipelas already possess early in the disease sufficient circulating antibody to neutralize 20 S.T.D. of filtrate per cubic centimeter of blood. The toxigenic activity of erysipelas streptococci, in most instances relatively poorly developed as compared with that of most scarlatinal streptococci, is probably counteracted at the 
site of the local lesion from the onset of the disease by the already existing antitoxic immunity. In the case of the few patients whose early sera failed to neutralize, antitoxin may have been present, but in amounts too small to be demonstrated by the method used. Four of these cases (Numbers 5, 10,17, and 19) failed to give positive skin reactions to 10 S.T.D. of filtrate. In fact, there are but three cases in the whole series (Numbers 24,25 , and 26) with positive skin tests and simultaneous absence of demonstrable antitoxin in the blood in the early acute stage of the disease, who would lend themselves to the support of a simple toxin-antitoxin concept of the disease, and even these fail through the apparent absence of antitoxin and the presence of positive skin tests (Number 26 not skin tested) about the end of the first week of convalescence.

The failure of the later convalescent sera to neutralize demands an explanation which at present, without further study, can be only hypothetical. It does not seem probable that this phenomenon is due to an actual disappearance of the neutralizing antibody from the blood, since this would be contrary to general immunological principles. It is suggested, therefore, that there appears in the blood of the patient an antibody to the absorbed products of the growth and dissolution of the streptococci distinct from the antitoxin, which under proper conditions will combine with similar products in culture filtrates to produce a demonstrable reaction. This antibody may tentatively be termed an antiallergen and the phase of immunity which it represents termed humoral allergy. On the basis of this hypothesis not only the apparent failure of the convalescent serum to neutralize the toxic action of the filtrate, but also the frequent occurrence of an intensified reaction might be explained as due to the reaction resulting from the combination of the antiallergen with bacterial products in the filtrate. Under these circumstances the passive neutralization of the toxin in the filtrate by antitoxin in the convalescent serum would be obscured and it would not be necessary to adopt the highly improbable view that antitoxin had disappeared from the blood in the convalescent stage of the disease.

Finally, it is necessary to attempt to correlate the results of the skin tests with those of the neutralization tests. Although the general trend of the two tests is in the same direction, it is obvious, as pointed 
out above, that they do not strictly parallel each other and that no simple relationship exists. It is suggested that a satisfactory correlation may be established by recognition of the probability that three immunity states-antitoxic immunity, tissue allergy, and humoral allergy-are concerned and that the time of appearance of each in measureable amount is variable and independent of the others. Such variation in the rate and development of immunity states and their mutual independence is well recognized. Besredka and Nakagawa (11) have stated that the cutaneous mechanism of defense is not, in general, associated with circulating antibodies. Swift, Derick and Hitchcock (12) have found that focal infections induce and maintain cutaneous reactivity, which may be diminished or destroyed by intra-

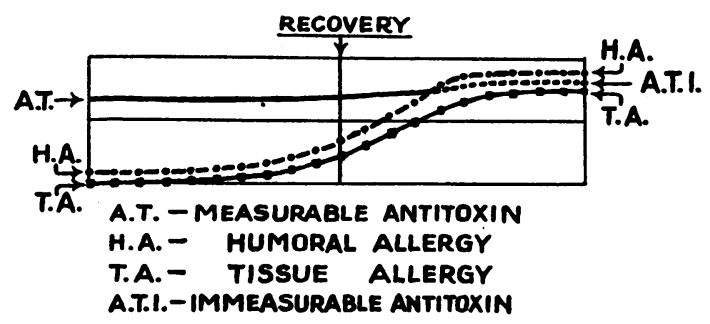

Fig. 1. Development of Immunity States in Erysipelas

At the point at which the line H.A. crosses the line A.T. the neutralization of toxin is obscured and the antitoxin becomes immeasurable.

venous injections of the organisms concerned. Gay and Rhodes (13) have reported that subcutaneous immunization may protect the skin against a lethal dose of the homologous organism, but not protect against an intravenous injection of a similar dose, and, conversely, that intravenous immunization may not protect against a lethal dose given subcutaneously.

If, then, it be assumed that the rate of development and degree of tissue allergy as measured by the skin tests, of antitoxic immunity as measured by the neutralization tests, and of humoral allergy as measured by the apparent loss of the neutralizing capacity of the serum, are variable and mutually independent, any variation in the results can be explained. To do so in each case would be impossible within the limits of this report. The most frequent relationship and 
general trend of the three immunity phases, however, are presented schematically in figure 1. Independent alterations in the slopes of the lines, which represent the rates of development of the three immunity phases, will serve to explain the different combinations of skin and neutralization tests at different stages of the disease in all of the patients studied.

\section{CONCLUSIONS}

As a result of the observations here presented it seems justifiable to conclude (1) that the pathogenesis of erysipelas is not comparable to the pathogenesis of the specific toxic phase of scarlet fever and (2) that the mechanism of recovery from erysipelas, in adults at least, is not a simple neutralization of a circulating toxin through the development of an antitoxin, but rather that it is more intimately related to the development of allergy to products of the growth and dissolution of streptococci in the erysipelatous lesion. Furthermore, the failure to find toxin in the blood and the usual presence of a neutralizing antibody in the early acute stage of the disease provide little basis for the view that antitoxin treatment in adults should be of particular value in erysipelas.

\section{BIBLIOGRAPHY}

1. Birkhaug, K. E., Bull. Johns Hopkins Hosp., 1925, xxxvi, 248. Studies on the Biology of the Streptococcus Erysipelatis. I. Agglutination and Agglutinin Absorption with the Streptococcus Erysipelatis.

2. Birkhaug, K. E., Bull. Johns Hopkins Hosp., 1925, xxxvii, 307. Studies on the Biology of the Streptococcus Erysipelatis. III. Experimental Production of Erysipelas in Rabbits and Demonstration of Protective Power of Immune Erysipelas Sera.

3. Birkhaug, K. E., Proc. Soc. Exp. Biol. and Med., 1925, xxiii, 201. Studies on the Biology of the Streptococcus Erysipelatis. IV. Toxin Production of the Streptococcus Erysipelatis.

4. Birkhaug, K. E., J. Am. Med. Assn., 1926, lxxxvi, 1411. Erysipelas. V. Observations on the Etiology and Treatment with Erysipelas Antistreptococcus Serum.

5. Birkhaug, K. E., J. Am. Med. Assn., 1927, lxxxviii, 885. Erysipelas. VI. Immunization with Soluble Toxin from Streptococcus Erysipelatis Against Recurrent Attacks of Erysipelas.

6. Singer, H. A., and Kaplan, B., J. Am. Med. Assn., 1926, 1xxxvii, 2141. Streptococcus Erysipelatis Toxin and Antitoxin. 
7. Dick, G. F., and Dick, G. H., J. Am. Med. Assn., 1924, lxxxii, 265. A Skin Test for Susceptibility to Scarlet Fever.

8. Dochez, A. R., Trans. Assoc. Amer. Phys., 1924, xxxix, 136. Studies in Scarlet Fever.

9. Blake, F. G., and Trask, J. D., J. Clin. Invest., 1926, iii, 397. Studies in Scarlet Fever. II. The Relation of the Specific Toxemia of Scarlet Fever to the Course of the Disease.

10. Dochez, A. R., and Stevens, F. A., J. Exp. Med., 1927, xlvi, 487. Studies on the Biology of Streptococcus. VII. Allergic Reactions with Strains from Erysipelas.

11. Besredka, A., and Nakagawa, S., Ann. Inst. Pasteur, 1927, xli, 607. Immunization Passive Contre le Tetanos par la Voie Cutanée.

12. Swift, H. F., Derick, C. L., and Hitchcock, C. H., J. Am. Med. Assn., 1928, xc, 906. Bacterial Allergy to Non-hemolytic Streptococci in its Relation to Rheumatic Fever.

13. Gay, F. P., and Rhodes, B., J. Inf. Dis., 1922, xxxi, 101. Experimental Erysipelas. Studies in Streptococcus Infection and Immunity. IV. 